\title{
ESTUDIO DE LA RESISTENCIA A LA CORROSION DEL ALUMINIO 6063 ANODIZADO
}

\section{STUDY OF CORROSION RESISTENCE OF ANODIZED ALUMINIUM 6063}

\author{
Karín Paucar Cuba ${ }^{1}$, Hugo Rojas Flores ${ }^{2}$, Abel Vergara Sotomayor ${ }^{3}$ \\ RESUMEN
}

\begin{abstract}
El estudio de la resistencia a la corrosión del anodizado de una aleación de aluminio (AA6063) en ácido sulfúrico a diferentes tiempos de anodizado: 30, 45 y 60 min. se realizó usando la espectroscopia de impedancia electroquímica (EIE) y el ensayo de niebla salina ácida. (ASTM B287). Los datos obtenidos por EIE y su correlación con los circuitos equivalentes más apropiados permitieron determinar los parámetros asociados a la capa porosa y a la capa barrera del óxido protector formado sobre la superficie del aluminio en estudio. La exposición de las muestras anodizadas durante $250 \mathrm{~h}$ a una niebla salina ácida permitió observar variaciones en su masa. De los resultados obtenidos por EIE y las pérdidas de masa de las muestras anodizadas se estableció que la película de anodizado de 45 minutos mostró una mayor resistencia a la corrosión en comparación con la obtenida a 60 y 30 min., respectivamente.
\end{abstract}

Palabras clave.- Aluminio, Anodizado, Impedancia electroquímica, Niebla salina ácida.

\begin{abstract}
The study of the corrosion resistance of anodized on aluminum alloy (AA6063) in sulfuric acid to different times: 30, 45 and $60 \mathrm{~min}$. was performed using electrochemical impedance spectroscopy (EIS) and the acid salt spray test (ASTM B287). The EIS'data and its correlation with the most appropriate equivalent circuits allowed to determine the parameters associated with the porous layer and the oxide layer protective barrier formed on the aluminum surface under study. Exposure of the samples anodized for a 250h salt spray acid allowed to observe changes in their mass. From the results obtained by EIS and the mass losses of the anodized samples was established that the anodized film of 45 minutes showed higher corrosion resistance compared to that obtained at 60 and 30 min, respectively.

Key words.- Aluminum, Anodized, Electrochemical impedance, Acid salt spray.
\end{abstract}

\section{INTRODUCCIÓN}

El aluminio y sus aleaciones son ampliamente utilizados a nivel industrial: construcción y edificación (paneles, techos, marcos, etc), contenedores y empaques, transporte (automóvil y aeroespacial), maquinarias y equipos [1]. Cuando son expuestos a la atmósfera sobre su superficie se forma, de manera natural, un óxido delgado de $\mathrm{Al}_{2} \mathrm{O}_{3}(2-4 \mathrm{~nm})$ [2] tenaz y de carácter protector que le aporta una excelente resistencia frente a la corrosión, especialmente en ciertos ambientes atmosféricos y químicos [3]. La posibilidad de producir mayores espesores de dicho oído a

\footnotetext{
${ }^{1}$ Ing. Docente investigador de la Facultad de Ingeniería Química y Textil, de la Universidad Nacional de Ingeniería. ${ }^{2}$ Ing. Docente investigador de la Facultad de Ingeniería Química y Textil, de la Universidad Nacional de Ingeniería. ${ }^{3}$ Ing. Docente Investigador de la Facultad de Ingeniería Química y Textil, de la Universidad Nacional de Ingeniería.
} 
partir del proceso electrolítico de anodizado se estableció en los años 1920 con la publicación de patentes utilizando electrolitos basados en ácido crómico, sulfúrico y oxálico [4]. Diferentes autores han realizado estudios sobre el efecto de las condiciones de anodizado en las cualidades protectoras de la película de óxido de aluminio obtenido electrolíticamente [5, 8]. La determinación de la resistencia a la corrosión de dicho óxido ha sido estudiada a través de ensayos de exposición natural [9], ensayos acelerados en cámara de niebla salina ácida [10] o ensayos electroquímicos [11].

La aplicación de la espectroscopia de impedancia electroquímica (EIE), para establecer la resistencia a la corrosión del óxido obtenido por anodizado del aluminio ha sido usada ampliamente $[12,15]$. Las cualidades de las denominadas capa porosa y capa barrera que componen el óxido de aluminio obtenido por anodizado y caracterizadas por los valores de $R_{p o}, C_{p o} y R_{b}, C_{b}$, respectivamente, puede ser obtenidos por circuitos eléctricos equivalentes que simulan la data de impedancia del óxido protector. En tal sentido, la aplicación de esta metodología para el estudio del comportamiento frente a la corrosión del anodizado del aluminio es una valiosa herramienta aplicable para el control de calidad in-situ del anodizado en curso.

En el presente trabajo se realizó el estudio de la resistencia a la corrosión usando la técnica EIE y el ensayo de niebla salina ácida del óxido de aluminio obtenido por el anodizado de una aleación AA6063 a partir de un baño de ácido sulfúrico a diferentes tiempos de anodizado.

\section{ANTECEDENTES DEL TRABAJO DE INVESTIGACION}

Las realización de la tesis de Maestría titulada: "Influencia de las variables de operación del proceso de anodizado del aluminio sobre el comportamiento anticorrosivo de la película de óxido de aluminio" desarrollada por el Ing. Abel
Vergara en la FIQT-UNI y del proyecto de investigación: "Evaluación de la influencia de la preparación de superficie en el comportamiento de los recubrimientos anticorrosivos mediante cámara de niebla salina" motivaron la investigación en el anodizado del aluminio y el artículo publicado por el grupo de Huang Y., Shih, H., con el título: "Evaluation of the resistance of anodized aluminum 6061 using electrochemical impedance spectroscopy (EIS), la realización del proyecto de investigación con los resultados que forman parte del presente artículo.

\section{PROCEDIMIENTO EXPERIMENTAL}

\section{Muestras de ensayo}

Se usaron pletinas de una aleación de aluminio AA6063 de uso comercial, cada muestra o probeta de ensayo se obtuvo del corte de pletinas de 50,0 x $11,0 \times 3,0 \mathrm{~mm}$, en la Tabla 1 se presenta la composición química de la aleación.

\section{Proceso de Anodizado}

Antes de ser anodizadas, las probetas se sometieron a un pretratamiento consistente en el desengrase/decapado con $\mathrm{NaOH}$ (5\% peso) y el neutralizado con $\mathrm{HNO}_{3}\left(60^{\circ} \mathrm{B}\right)$ y posterior pesado en una balanza Sartoriuos TE214S. El proceso de anodizado se realizó por inmersión en una cuba electrolítica conteniendo una solución de ácido sulfúrico (96,7-97,5\% de pureza) al $15 \%$ en peso, $15-17 \mathrm{~V}, 1,5 \mathrm{~A} / \mathrm{dm}^{2}$ y una temperatura de anodizado de $18 \pm 2{ }^{\circ} \mathrm{C}$. Los tiempos de anodizado de las probetas fueron: 30,45 y $60 \mathrm{~min}$. El sellado de las mismas se realizó en agua hirviendo durante $30 \mathrm{~min}$. Finalmente, las probetas anodizadas y selladas nuevamente se pesaron. La medición del espesor de la película de óxido de aluminio obtenido se realizó con ayuda del medidor de espesor BYKO-TEST7500 y siguiendo la norma ASTM D1400 [16] y ASTM B244 [17].

En la Figura 1 se muestra el proceso de anodizado de las probetas de ensayo. 


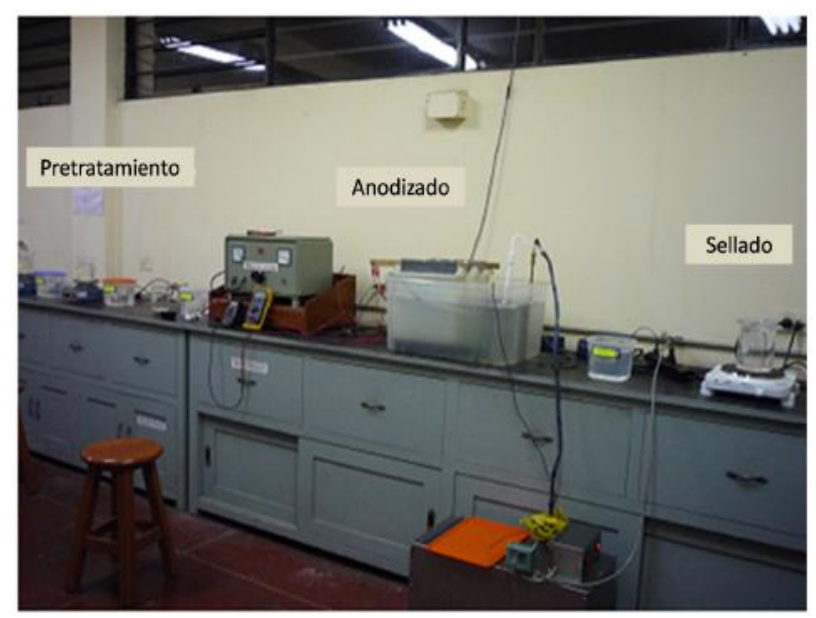

Fig. 1 Esquema del proceso de anodizado.

Tabla 1. Composición química de la aleación AA6063 (\%peso).

\begin{tabular}{|c|c|c|c|c|c|c|c|c|c|}
\hline $\mathrm{Mg}$ & $\mathrm{Si}$ & $\mathrm{Fe}$ & $\mathrm{Cu}$ & $\mathrm{Mn}$ & $\mathrm{Zn}$ & $\mathrm{Cr}$ & $\mathrm{Ti}$ & Otros & $\mathrm{Al}$ \\
\hline $\begin{array}{l}0,45- \\
0,90\end{array}$ & $\begin{array}{l}0,20- \\
0,60\end{array}$ & 0,35 & 0,10 & 0,10 & 0,10 & 0,10 & 0,10 & 0,10 & balance \\
\hline
\end{tabular}

\section{Evaluación del comportamiento frente a la} corrosión

Ensayos Electroquímicos.- Los espectros de impedancia electroquímica se obtuvieron a partir de la medición de la impedancia utilizando un Potenciostato/Galvanostato/ZFRA, Reference 600 de Gamry Instruments y una celda de medición constituida por un tubo de vidrio, una base de apoyo para la probeta de estudio, una pinza de acero inoxidable para sujeción de la probeta al tubo y la base, un electrodo de referencia Gamry de Calomel saturado (ECS) y un electrodo de alambre de platino (Figura 2).

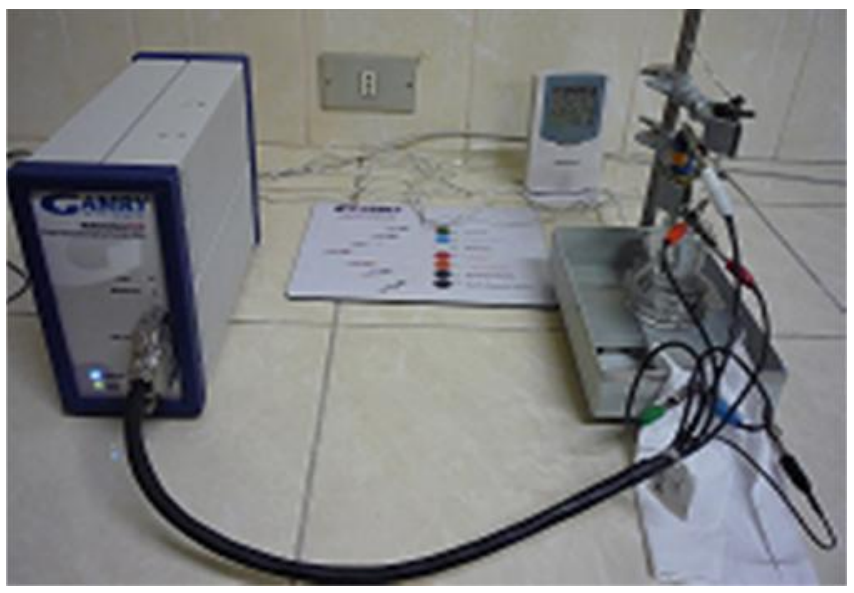

Fig. 2 Equipo y celda de medición de EIE.

El área de exposición de la probeta al electrolito de ensayo se obtuvo con la ayuda de pegatinas de plástico de 5,5 × 5,5 cm con un área libre de 3,14 $\mathrm{cm}^{2}$ y las cuales se pegaron sobre las probetas anodizadas antes de realizar el ensayo. 
Como electrolito de ensayo se utilizó una solución acuosa de cloruro de sodio (SCHARLAU, 99,8\% de pureza) $0,5 \mathrm{M}$ y $\mathrm{pH}$ 6,5-7,2.

Luego de una hora de inmersión, las impedancias electroquímicas se midieron en el rango de frecuencias de $10^{5}-10^{-3} \mathrm{~Hz}$. y con una amplitud de $10 \mathrm{mV}$, las mediciones se realizaron por triplicado.

Ensayo de niebla salina ácida.- La exposición de las probetas en una cámara de niebla salina ácida se realizó por triplicado y siguiendo el procedimiento descrito en la norma ASTM B 287 [16]. En una cámara de niebla BASS de $1 \mathrm{~m}^{3}$ de capacidad (Figura 3) y cuyas condiciones principales se describe a continuación:

Solución salina $\mathrm{NaCl}$ : $5 \%$ en peso y $\mathrm{pH}$ 2-4 usando ácido acético.

Temperatura en el interior de la cámara de ensayo: $35 \pm 1{ }^{\circ} \mathrm{C}$.

Presión de aire: 1 bar.

Volumen de niebla: $1,2 \mathrm{~mL} / \mathrm{cm}^{2} \cdot \mathrm{h}$.

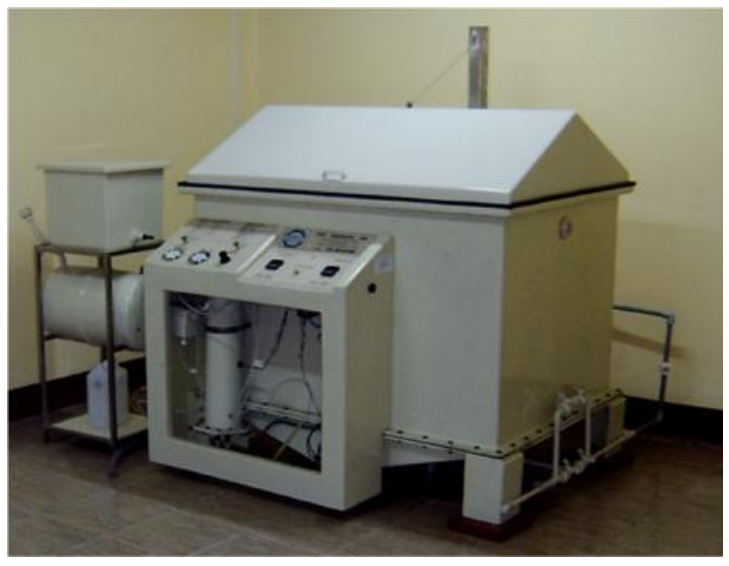

Fig. 3 Cámara de niebla salina, BASS.

Antes de realizar la exposición de las probetas anodizadas a la niebla por $250 \mathrm{~h}$ se procedió:

$\checkmark$ Aislamiento de los bordes con cinta aislante.

$\checkmark$ Aislamiento de agujeros de sujeción con masilla.

$\checkmark \quad$ Limpieza y desengrase con agua.

$\checkmark \quad$ Corte o incisión en forma de aspa en la parte inferior de las probetas.

Al término del ensayo se procedió al lavado y secado de las probetas de ensayo así como al pesado de las mismas.

\section{RESULTADOS Y DISCUSIÓN}

\section{Muestras anodizadas}

En la Tabla 2 se reporta las masas promedio de las probetas de ensayo antes y después del proceso de anodizado y sellado así como el espesor promedio de las probetas para los diferentes tiempos de anodizado: 30, 45 y 60 min.
Tabla 2. Masas de las probetas antes y después del anodizado y espesor promedio del óxido.

\begin{tabular}{llll}
\hline & $\begin{array}{l}\text { Masa } \\
\text { promedio } \\
\text { antes de de } \\
\text { anodizado } \\
(\mathrm{g})\end{array}$ & $\begin{array}{l}\text { Masa } \\
\text { promedio } \\
\text { después de } \\
\text { anodizado }\end{array}$ & $\begin{array}{l}\text { Espesor } \\
\text { promedio } \\
(\mathrm{g})\end{array}$ \\
& $\begin{array}{l}\text { (mils) } \\
\text { (min) }\end{array}$ & \\
\hline 30 & 43,6906 & 43,5203 & 0,486 \\
45 & 42,3818 & 42,3027 & 0,704 \\
60 & 43,4691 & 43,4296 & 0,940 \\
\hline
\end{tabular}

\section{Espectroscopia de impedancia electroquímica (EIE)}

En las (Figuras $4 \mathrm{a}$ y b) se muestran los Diagramas de Bode ( $Z$ vs. $f$ ) y Nyquist (-Z" vs. $Z^{\prime}$ ), respectivamente obtenidos para los tiempos de anodizado en estudio: 30, 45 y $60 \mathrm{~min}$. 
A altas frecuencias, en el Diagrama de Bode de la Figura 4a se observa que la impedancia del óxido protector, Z, para 45 y 60 min. de anodizado es de 1-1,5 órdenes de magnitud mayor al obtenido para $30 \mathrm{~min}$.

Las impedancias del óxido para 45 y $60 \mathrm{~min}$. de anodizado, muestran la formación de una sección horizontal con valores de impedancia comprendidos entre $10^{4}-10^{5} \Omega \cdot \mathrm{cm}^{2}$, siendo mayor la obtenida para $45 \mathrm{~min}$. en comparación al obtenido a $60 \mathrm{~min}$.

Ello puede estar atribuido al ataque de la película óxido debido al mayor tiempo de exposición al electrolito ácido usado para el anodizado.

A bajas frecuencias, los valores de impedancia según el tiempo de anodizado empleado, no muestran diferencias apreciables.

Los diagramas de Nyquist mostrados en la Figura $4 b$ ), permiten observar un comportamiento y valores de impedancia muy parecidos en los tres tiempo de anodizado en estudio, con lo cual se puede indicar que este diagrama es menos sensible a mostrar el comportamiento del óxido durante en los primeros instantes de inmersión en el electrolito de ensayo (1h).
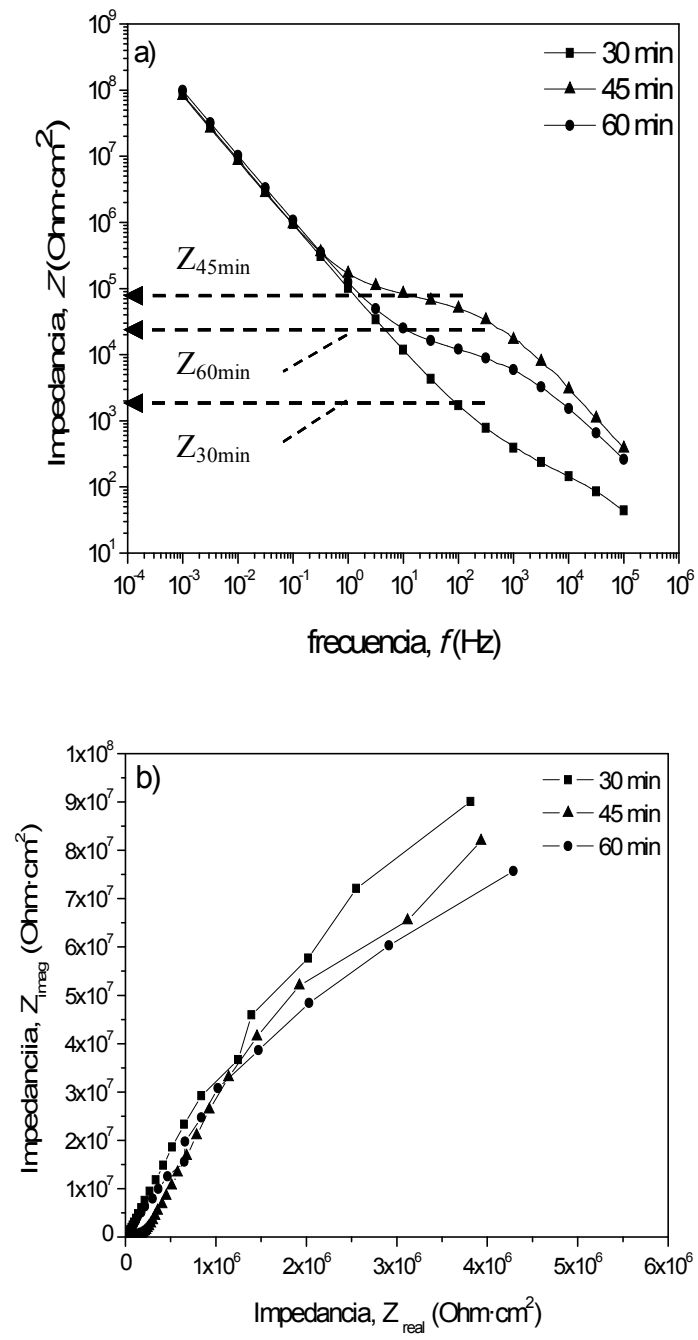

Fig. 4 a) Diagramas de Bode y b) diagramas de Nyquist para 30, 45 y $60 \mathrm{~min}$. de anodizado.

Los parámetros concernientes a la capa porosa $\left(\mathrm{C}_{\mathrm{po}}\right.$ y $\left.R_{p o}\right)$ y la capa barrera $\left(C_{b}\right.$ y $\left.R_{b}\right)$ a partir de la data de impedancia obtenida experimentalmente para cada uno de los tiempos de anodizado en estudio, fueron simulados a través de un circuito eléctrico equivalente que simula la capa de óxido de aluminio [15], en la Figura 5 se presenta el circuito de Randles propuesto para las condiciones de anodizado en estudio.

La designación de cada uno de los elementos del circuito equivalente es como se indica: 
$\mathrm{R}_{\mathrm{e}}$ : resistencia del electrolito, $\Omega \cdot \mathrm{cm}^{2}$

$\mathrm{C}_{\mathrm{po}}$ : capacitancia de la capa porosa, $\mu \mathrm{F} \cdot \mathrm{cm}^{-2}$

$\mathrm{R}_{\mathrm{po}}:$ resistencia de la capa porosa, $\Omega \cdot \mathrm{cm}^{2}$

$\mathrm{C}_{\mathrm{b}}$ : capacitancia de la capa barrera, $\mu \mathrm{F} \cdot \mathrm{cm}^{-2}$

$\mathrm{R}_{\mathrm{b}}$ : resistencia de la capa barrera, $\Omega \cdot \mathrm{cm}^{2}$

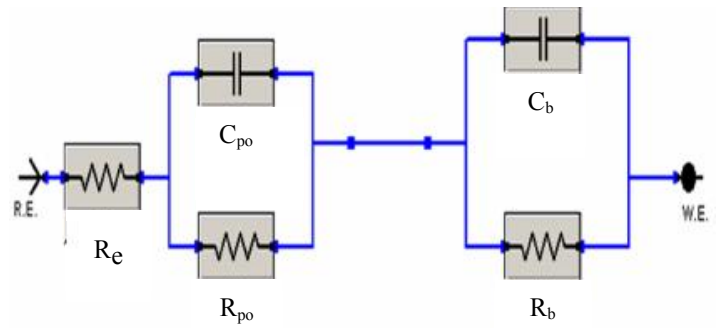

Fig. 5 Circuito Randles del anodizado del aluminio AA6063.

De los valores obtenidos para los parámetros $\mathrm{C}_{\mathrm{b}} \mathrm{y}$ $\mathrm{R}_{\mathrm{b}}$ correspondientes a la capacitancia y resistencia de la capa barrera, respectivamente, del óxido protector obtenido a diferentes tiempos de anodizado, se observa que para el tiempo de anodizado de $45 \mathrm{~min}$. los valores de dichos parámetros son los más altos comparativamente, lo cual permitirá que la capa barrera ofrezca una alta capacidad protectora frente a la corrosión del óxido anodizado tras el paso del electrolito a través de la capa porosa. En la Tabla 3 se muestra los valores de $R_{p o}, R_{b}, C_{p o}$ y $C_{b}$ obtenidos para los diferentes tiempos de anodizado.

Tabla 3. Parámetros del circuito Randles del anodizado del aluminio AA6063.

\begin{tabular}{lllll}
\hline $\mathrm{T}$ & $\begin{array}{l}\mathrm{C}_{\mathrm{po}} \\
\left(\mu \mathrm{F} \cdot \mathrm{cm}^{-}\right.\end{array}$ & $\begin{array}{l}\mathrm{R}_{\mathrm{po}} \\
\left(\Omega \cdot \mathrm{cm}^{2}\right)\end{array}$ & $\begin{array}{l}\mathrm{C}_{\mathrm{b}} \\
\left(\mu \mathrm{F} / \mathrm{cm}^{2}\right)\end{array}$ & $\begin{array}{l}\mathrm{R}_{\mathrm{b}} \\
\left(\Omega \cdot \mathrm{cm}^{2}\right)\end{array}$ \\
\hline $\min )$ & $\left.{ }^{2}\right)$ & & & \\
30 & 1,562 & $1,25 \cdot 10^{9}$ & 0,107 & $0,27 \cdot 10^{3}$ \\
45 & 1.405 & $0,80 \cdot 10^{9}$ & 0.035 & $1,68 \cdot 10^{3}$ \\
60 & 1,437 & $1,14 \cdot 10^{9}$ & 0,037 & $1,42 \cdot 10^{3}$ \\
\hline
\end{tabular}

Comparando los valores de $\quad \mathrm{C}_{\mathrm{po}} \quad \mathrm{y} \quad \mathrm{R}_{\mathrm{po}}$ correspondientes a la capacitancia y resistencia de la capa porosa del óxido protector obtenido a diferentes tiempos de anodizado, se observa que para el tiempo de anodizado de $45 \mathrm{~min}$. la capacitancia $\mathrm{C}_{\mathrm{po}}$ es ligeramente menor al mostrado para 30 y $60 \mathrm{~min}$., lo cual está asociado a una menor porosidad del óxido a estas condiciones de anodizado [15,19], mientras que el valor de $R_{p o}$ resulta ser ligeramente menor, lo cual podría estar relacionado con el diámetro, profundidad y calidad del sellado del poro [14] y por tanto una ligeramente menor resistencia a la penetración del electrolito.

De los valores de $R_{p o}, C_{p o}$ y $R_{b}, C_{b}$ se puede establecer desde el punto de vista de resistencia contra la corrosión, $R$, en función del tiempo de anodizado de aluminio, el siguiente orden de mayor a menor resistencia:

$$
R_{\mathrm{T}=45 \text { min. }}>R_{\mathrm{T}=60 \text { min. }}>R_{\mathrm{T}=30 \text { min. }}
$$

\section{Niebla salina ácida}

Las probetas de aluminio anodizado fueron expuestas por triplicado a la niebla salina ácida según procedimiento descrito en la norma ASTM B287, el tiempo de exposición fue de $250 \mathrm{~h}$ a fin de poder observar alguna diferencia en su comportamiento frente a la corrosión al menor tiempo de ensayo posible, dada las condiciones de alta agresividad del medio de exposición en la cámara.

Al término del ensayo las muestras fueron lavadas, secadas y pesadas a fin de poder comparar el grado de resistencia a la corrosión que ofrecieron a diferentes espesores de anodizado. Los resultados de pérdida de masa se describen en la Tabla 4.

Tabla 4. Pérdidas de masa de probetas anodizadas y selladas a diferentes tiempos de anodizado después de $250 h$ de ensayo.

\begin{tabular}{cc}
\hline $\begin{array}{c}\mathrm{T} \\
(\mathrm{min})\end{array}$ & $\begin{array}{c}\text { Pérdidas de masa } \\
\text { promedio }(\mathrm{g})\end{array}$ \\
\hline 30 & 0,0013 \\
45 & 0,0007 \\
60 & 0,0011 \\
\hline
\end{tabular}


Las muestras de aluminio anodizadas y selladas para una exposición de $250 \mathrm{~h}$ en niebla salina ácida mostraron pérdidas de masa mensurables aunque con una incipiente evidencia visual del deterioro. Los valores de pérdida de masa para los diferentes tiempo de anodizado, permitieron establecer un mayor grado de corrosión para las muestras de aluminio anodizadas durante $30 \mathrm{~min}$. en comparación a aquellas anodizadas a 60 y $45 \mathrm{~min}$., respectivamente.

\section{CONCLUSIONES}

Los espectros de impedancia típicos para los diferentes tiempos de anodizado estudiados, nos muestran diferencias apreciables en el rango de frecuencias estudiadas, haciendo de la EIE una herramienta sensible en la detección de cambios en las características de la capa porosa y de la capa barrera en función del tiempo de anodizado.

Para los diferentes tiempos de anodizado, la capacitancia de la capa porosa es hasta 10 veces mayor que la capacitancia de la barrera, con lo cual, se puede verificar una mayor permeabilidad del electrolito en la capa porosa que en la capa barrera, siendo esta última la que finalmente protegerá al sustrato metálico contra la corrosión.

Los valores de $C_{p o}, R_{p o}$ y $C_{b}, R_{b}$ obtenidos para el circuito equivalente propuesto, muestran en general, que la mayor resistencia frente a la corrosión lo experimenta la probeta de aluminio con 45 min. de anodizado y que un comportamiento ligeramente parecido se observa para el tiempo de anodizado de $60 \mathrm{~min}$.

Un comportamiento prácticamente deficiente (respecto a la resistencia de la capa barrera $\mathrm{R}_{\mathrm{b}}$ ) frente a los anteriores lo muestra el tiempo de anodizado de $30 \mathrm{~min}$. Desde el punto de vista del consumo de energía en un proceso de anodizado, también resultaría económicamente más eficiente el proceso de anodizado a $45 \mathrm{~min}$.

El estudio del comportamiento frente a la corrosión del aluminio anodizado ha podido llevarse a cabo aplicando la técnica de espectroscopia de impedancia electroquímica (EIS), la cual se convierte en una herramienta muy útil para el estudio de este tipo de recubrimientos.
El ensayo de niebla salina ácida para un tiempo de exposición de $250 \mathrm{~h}$ resulta ser un ensayo que permite obtener información comparativa sobre el grado de resistencia a la corrosión de muestras anodizadas.

Los resultados obtenidos por los ensayos de niebla salina ácida coinciden con los obtenidos a través de los ensayos electroquímicos realizados, con lo cual, por los resultados de ambos ensayos se puede establecer el siguiente orden creciente de resistencia a la corrosión dentro de los tres tiempos de anodizado:

$$
\mathrm{R}_{\mathrm{T}=45 \text { min. }}<\mathrm{R}_{\mathrm{T}=60 \text { min. }}<\mathrm{R}_{\mathrm{T}=30 \text { min. }}
$$

\section{AGRADECIMIENTOS}

Los autores agradecen al Instituto de Investigación de la FIQT y al IGI-UNI por el financiamiento y adquisición del Potenciostato/Galvanostato/ZFRA incluidos los softwares, así como a los alumnos de los diferentes ciclos de estudios de nuestra facultad y sus egresados, quienes hicieron posible la implementación y acondicionamiento de un nuevo ambiente en el Gabinete de Corrosión de la FIQT para la realización de proyectos de investigación relacionados con el estudio del anodizado del aluminio.

\section{REFERENCIAS}

1. Ashby, M. F., Jones, D. R., "Engineering Materials", vol. 2, 2da. edición, ButterworthHeinemann, (1998) 101.

2. Safrany Pechiney, J. S., "Anodisation de l'aluminium et de ses alliages", Centre de Recherches de Voreppe, 1630 (2001) pp. 126.

3. Campbell, F. C., "Manufacturing Technology for Aerospace Structural Materials, 1ra. edición, Elsevier, (2006)15-90.

4. Brace, A., "The technology of Anodizing Aluminium", 3ra. edición, (2000) 2.

5. Habazaki, H., et al., "Nanoscale enrichments of substrate elements in the growth of thin oxide films", Revista Corrosion Science, N³9, pp. 731-737, (1997).

6. Diggle, J. W., "Oxide and Oxide Films", vol. 2, Marcel Dekker Inc., N. York 1973.

7. De Laet, J., Terryn, H., Vereecken, J., "Development of an optical model for steady 
state porous anodic films on aluminium formed in phosphoric acid", Revista Thin Solid Films, N³20, pp. 241-252, (1998).

8. Kim, Y. C., Quint, B., Kesler, R. W., Oelkurg, D., "Structructural properties of electrochemically designed porous oxide films on AlMg1", Revista Journal of Electroanalytical Chemistry, $\mathrm{N}^{\circ} 468$, pp. 121126, (1999).

9. Feliu, S. Jr., González, J. A., López, E. V., "Characterisation of porous and barrier layers of anodic oxides on different aluminium alloys". Revista Journal of Applied Electrochemistry, $\mathrm{N}^{\circ} 37$, pp. 1027-1037, (2007).

10. ASESAN., "Directrices de la Marca de Calidad de QUALANOD para el Anodizado del Aluminio en medio sulfúrico", España (2004) 11.

11. González Fernández, J. A., "Control de la Corrosión. Estudio y medidas por Técnicas Electroquímicas". CENIM, Madrid 1989.

12. Hitzig, J., Jeuttner, K., "AC- impedance mesurments on porous aluminium oxide films”, Revista Corrosion Science, N`24, pp. 945-952, (1984).

13. Mansfeld, F., Kendig, M. W., "Evaluation of Anodized Aluminum Surfaces with Electrochemical Impedance Spectroscopy", Revista Journal of the Electrochemical Society, $\mathrm{N}^{\circ} 135$, pp. 828-833, (1988).

14. Moutarlier, V., Gigandet, M. P. , Normand B., "EIS Characterization of anodic films formed on 2024 aluminium alloy, in sulfuric acid containing molybdate or permanganate species", Revista Corrosion Science, $\mathrm{N}^{\circ} 47$, pp. 937-951, (2005).

15. Huang, Y., Shih, H., "Evaluation of the corrosion resistance of anodized aluminum 6061 using electrochemical impedance spectroscopy (EIS)", Revista Corrosion Science, $N^{\circ} 50$, pp. 3569-3575, (2008).

16. Norma ASTM D 1400., "Standard Test Method for Nondestructive Measurement of Dry Film Thickness of Nonconductive Coatings Applied to a Nonferrous Metal Base”, ASTM International, Filadelfia 2000.

17. Norma ASTM B 244., "Standard Test Method for Measurement of Thickness of Anodic Coatings on Aluminum and of Other Nonconductive Coatings on Nonmagnetic Basis Metals with Eddy-Current Instruments", ASTM International, Filadelfia 2009.

18. Norma ASTM B 287., "Standard Method of Acetic Acid-Salt Spray (Fog) Testing", ASTM International, Filadelfia 1987.

19. Sullivan, P. O, Wood, G. C., "The morphology and mechanism of formations of porous anodic films on aluminium", Revista Proceedings of the Royal Society A, N 317 , pp. 511-543, (1970).

Correspondencia: paucar@uni.edu.pe

Recepción de originales: diciembre 2012

Aceptación de originales: abril 2013 